\title{
Variation of aflatoxin M1 contamination in milk and milk products collected during winter and summer seasons
}

\begin{abstract}
Total 221 samples of milk and milk products were collected during winter (November 2011February 2012) and 212 samples were collected during summer (May-August 2012) from central areas of Punjab, Pakistan. The samples were analyzed for the presence of aflatoxin M1 (AFM1) with a validated HPLC method equipped with florescence detector. The results revealed that from winter season almost $45 \%$ samples of milk and milk products were found to be contaminated with AFM1 i.e. $40 \%$ of raw milk, $51 \%$ of UHT milk, $37 \%$ of yogurt, $60 \%$ of butter and $43 \%$ of ice cream samples and 27,24, 25, 34 and $17 \%$ of samples were found above the recommended limit for AFM1, respectively. However, from summer season 32\% samples of milk and milk products were found to be contaminated i.e. $36 \%$ of raw milk, $31 \%$ of UHT milk, $29 \%$ of yogurt, $40 \%$ of butter and $24 \%$ of ice cream and 23, 23, 18, 20 and 5\% of samples were found above the permissible limit for AFM1, respectively. The levels of contamination in winter milk and milk product samples were significantly higher $(\alpha \leq 0.05)$ than in summer season. The occurrence of AFM1 in milk and milk products were higher, demanding to implement strict regulations and also urged the need for continuous monitoring of milk and milk products in order to minimize the health hazards.
\end{abstract}

Keyword: Aflatoxin M1; Milk and milk products; HPLC; Seasonal variations 